\title{
Comparison of neuronal death and expression of TNF- $\alpha$ and MCT4 in the gerbil hippocampal CA1 region induced by ischemia/reperfusion under hyperthermia to those under normothermia
}

\author{
TAEK GEUN OHK ${ }^{1 *}$, JI HYEON AHN ${ }^{2,3 *}$, YOUNG EUN PARK ${ }^{3}$, TAE-KYEONG LEE $^{2}$, BORA KIM $^{3}$, \\ JAE-CHUL LEE ${ }^{3}$, JUN HWI CHO ${ }^{1}$, JOON HA PARK ${ }^{4}$, MOO-HO WON ${ }^{3}$ and CHOONG-HYUN LEE ${ }^{5}$ \\ ${ }^{1}$ Department of Emergency Medicine, and Institute of Medical Sciences, Kangwon National University Hospital, School of \\ Medicine, Kangwon National University, Chuncheon, Gangwon 24289; ${ }^{2}$ Department of Biomedical Science, Research \\ Institute of Bioscience and Biotechnology, Hallym University, Chuncheon, Gangwon 24252; ${ }^{3}$ Department of Neurobiology, \\ School of Medicine, Kangwon National University, Chuncheon, Gangwon 24341; ${ }^{4}$ Department of Anatomy, College of \\ Korean Medicine, Dongguk University, Gyeongju, Gyeongbuk 38066; ${ }^{5}$ Department of Pharmacy, College of \\ Pharmacy, Dankook University, Cheonan, Chungcheongnam 31116, Republic of Korea
}

Received February 12,2020; Accepted May 12, 2020

DOI: $10.3892 / \mathrm{mmr} .2020 .11182$

\begin{abstract}
Monocarboxylate transporter 4 (MCT4) is a high-capacity lactate transporter in cells and the alteration in MCT4 expression harms cellular survival. The present study investigated whether hypothermia affects tumor necrosis factor- $\alpha$ (TNF- $\alpha$ ) and MCT4 immunoreactivity in the subfield cornu ammonis 1 (CA1) following cerebral ischemia/reperfusion (IR) in gerbils. Hypothermia was induced for $30 \mathrm{~min}$ before and during ischemia. It was found that IR-induced death of pyramidal neurons was markedly augmented and occurred faster under hyperthermia than under normothermia. TNF- $\alpha$ immunoreactivity in the pyramidal cells started to increase at $3 \mathrm{~h}$ after IR and peaked at 1 day after IR under normothermia. However, in hyperthermic control and sham operated gerbils, TNF- $\alpha$ immunoreactivity was significantly increased compared
\end{abstract}

Correspondence to: Professor Moo-Ho Won, Department of Neurobiology, School of Medicine, Kangwon National University, 1 Gangwondaehak-gil, Chuncheon, Gangwon 24341, Republic of Korea

E-mail: mhwon@kangwon.ac.kr

Professor Choong-Hyun Lee, Department of Pharmacy, College of Pharmacy, Dankook University, 119 Dandae-ro, Cheonan, Chungcheongnam 31116, Republic of Korea

E-mail: anaphy@dankook.ac.kr

*Contributed equally

Key words: hippocampal CA1 region, hyperthermic condition, monocarboxylate transporter 4 , neuronal death, transient forebrain ischemia with the normothermic gerbils, and IR under hyperthermia caused a more rapid and significant increase in TNF- $\alpha$ immunoreactivity in pyramidal neurons than under normothermia. In addition, in the normothermic gerbils, MCT4 immunoreactivity began to decrease in pyramidal neurons from $3 \mathrm{~h}$ after IR and markedly increased at 1 and 2 days after IR. On the other hand, MCT4 immunoreactivity in pyramidal neurons of the hyperthermic gerbils was significantly increased from $3 \mathrm{~h}$ after IR, maintained until 1 day after IR and markedly decreased at 2 days after IR. These results indicate that acceleration of IR-induced neuronal death under hyperthermia might be closely associated with early alteration of TNF- $\alpha$ and MCT4 protein expression in the gerbil hippocampus after IR.

\section{Introduction}

Ischemia/reperfusion (IR) in the brain causes selective neuronal death in vulnerable brain structure, such as the hippocampus; especially, among neurons in the hippocampus, pyramidal neurons of cornu ammonis 1 (CA1) are the most vulnerable after IR $(1,2)$. IR-induced death of CA1 pyramidal neurons occurs a few days after IR, and this phenomenon is called 'delayed neuronal death (DND)' (1). Several factors, such as age and sex, can affect the degree of neuronal damage in the brain (3-5). Among them, brain and body temperature have been hypothesized to be a major factor in neuronal survival and death after IR injury (6-9).

Elevated temperature is closely related to neurological deficits, enlargement of ischemic lesions, microvascular injury and increase in neuronal damage in various rodent models of brain ischemia (10-14). Hyperthermia increases the metabolic rate (15), which is harmful to the ischemic brain as there is an imbalance between energy supply and demand during ischemia $(7,16)$. 
Metabolic adaptation, which is a switch between oxidative and glycolytic metabolism, occurs during ischemia and after reperfusion in brains (17). Such metabolic changes increase the production and accumulation of lactates in ischemic tissues $(18,19)$. Lactate trafficking between cells is facilitated mainly by proton-dependent symporters known as monocarboxylate transporters (MCTs). Currently, 14 isoforms of the MCT family are known, but only three MCTs, MCT1, MCT2 and MCT4, have been elucidated as lactate transporters in the central nervous system (20). Among them, MCT4 is known to be expressed in neurons and/or astrocytes (21-24).

Our previous studies reported the effects of hyperthermia on hippocampal neuronal damage after IR in gerbils $(8,9)$. In addition, a chronological change in MCT4 protein expression in the gerbil hippocampus after IR was revealed under normothermia $(21,24)$. However, to the best of our knowledge, there have been no studies on changes of MCT protein expression levels in the hippocampus under hyperthermia before and/or during ischemic insults. Therefore, the present study compared MCT4 and TNF- $\alpha$ immunoreactivities in IR-induced CA1 under hyperthermic condition to that under normothermic condition.

\section{Materials and methods}

Experimental animals. Male Mongolian gerbils (Meriones unguiculatus; weight, 66-74 g; age, 6 months) were obtained from the Experimental Animal Center, Kangwon National University, Chuncheon, Republic of Korea. Gerbils were housed under conventional housing conditions at $23 \pm 3^{\circ} \mathrm{C}$ with relative humidity of $55 \pm 5 \%$, under a 12 -h light/dark cycle. Free access to food and water was allowed. Experimental procedures for this study were approved by the Institutional Animal Care and Use Committee at Kangwon National University (approval no. KW-200113-1).

The process of handling and caring animals conformed to the guidelines of the current international laws and policies $(25,26)$. The numbers of gerbils used in this study and the suffering caused by the procedures used in all experiments was minimized. Body weight and behavior of all animals were monitored every other day. Humane endpoints were determined when the animals showed weight loss $>20 \%$, dehydration, or loss of ability to ambulate. No animals showed signs of humane endpoints intended to be euthanized immediately. The method of euthanasia was chemical and physical method. After each animal was profoundly anesthetized using $60 \mathrm{mg} / \mathrm{kg}$ of pentobarbital sodium, and cardiac perfusion was conducted. Confirmation of death was evaluated with vital signs including heart beats, pupillary response, and respiratory pattern (lack of cardiac activity for $5 \mathrm{~min}$ through cardiac palpation, unresponsiveness to light with dilated pupils using light into the eyes of the animal and lack of spontaneously breathing pattern with shallow and irregular breathing pattern).

Experimental groups and induction of IR. Experimental animals (total $\mathrm{n}=109$ ) were divided into seven groups: i) Normal animals (normal group; $n=5$ ); ii) control animals with normothermia (NT/control group; $n=5)$; iii) sham-operated animals with normothermia (NT/sham group; $n=5)$; iv) IR-operated animals with normothermia (NT/IR group; $\mathrm{n}=7$ at each time point); v) control animals with hyperthermia (HT/control group; $n=5)$; vi) sham-operated animals with hyperthermia (HT/sham group; $n=5)$; and vii) IR-operated animals with hyperthermia (HT/IR group; $n=7$ at each time point). Since there were no significant differences between the normal group and NT/control group in the present study, data of the normal group are not shown.

The gerbils in each group were anesthetized with a mixture of $2.5 \%$ isoflurane (Baxtor) in $30 \%$ oxygen and $70 \%$ nitrous oxide using inhalation anesthesia equipment (Harvard Apparatus), with a modification of methods of previous studies (27-29); inhalation anesthetics for the gerbils maintained precise control over the dosage of the anesthetic agent and enabled them to rapidly recover (30). Hyperthermia was induced by exposing the gerbils to a heating pad (homeothermic monitoring system, Harvard Apparatus) connected to a rectal thermistor under anesthesia until their rectal temperature was elevated to $39.5 \pm 0.2^{\circ} \mathrm{C}$, and the animals were maintained at this temperature for $30 \mathrm{~min}$ before and during the surgery. For normothermic condition, the rectal temperature was controlled at $37.0 \pm 0.5^{\circ} \mathrm{C}$. For the induction of IR, as previously described (9), both common carotid arteries were isolated and occluded by using non-traumatic aneurysm clips for $5 \mathrm{~min}$. Then, the animals were maintained in thermal incubators (temperature $23^{\circ} \mathrm{C}$; humidity $60 \%$ ) to maintain body temperature at a normothermic level until they were euthanized (Fig. 1). The animals in the NT/sham and HT/sham groups were exposed to the same surgical processes without bilateral carotid artery occlusion.

Tissue processing for histology. The animals of the NT/IR and HT/IR groups were sacrificed, and their brain sections containing the hippocampus were prepared at designated times ( 3 h, 12 h, 1 day, 2 days, 3 days and 5 days after IR). To reduce the number of animals, the brain sections of the NT/control, NT/sham, HT/control and HT/sham groups were obtained only at 5 days after sham operation. For preparation of sections, as previously described $(9,21)$, the animals were perfused transcardially with solution of $4 \%$ paraformaldehyde after checking vital signs to ensure that the animals were profoundly anesthetized with $60 \mathrm{mg} / \mathrm{kg}$ pentobarbital sodium (JW Pharmaceutical Co., Ltd.) (31). Their brains were removed and post-fixed in the same fixative at room temperature for $6 \mathrm{~h}$. The brain tissues were cryoprotected with solution of $30 \%$ sucrose, and the frozen tissues were serially sectioned into $30-\mu \mathrm{m}$ coronal sections.

Fluoro-Jade B (FJB) histofluorescence staining. FJB is a fluorescent derivative used for histological staining of degenerating neurons. In the present study, FJB histofluorescence staining was conducted to examine neuronal damage and death in the hippocampus after IR. As described previously $(9,21,32)$, the tissues were immersed in a solution of $0.06 \%$ potassium permanganate and stained with a solution of $0.0004 \%$ FJB (Histochem) for $45 \mathrm{~min}$ at room temperature.

To analyze the numbers of damaged (dead) neurons, the stained sections were observed with an epifluorescent microscope at magnification, x20 (Carl Zeiss AG). According to our previously published method (21), FJB-positive cells were examined with an epifluorescent microscope (Carl Zeiss AG) 
equipped with 450-490 $\mathrm{nm}$ of blue excitation light and a barrier filter. This microscope was equipped with a digital camera connected to a PC monitor. Digital images of FJB-positive cells in the same areas in the hippocampus were captured. These captured cells were counted using an image analyzing software (Optimas v6.5; CyberMetrics)

Immunohistochemistry. Immunohistochemical staining was performed to examine changes in MCT4 expression in the hippocampus after IR according to our previously published method $(9,21,32)$. Briefly, the sections $(30-\mu \mathrm{m})$ were treated with a solution of $0.3 \%$ hydrogen peroxide for $20 \mathrm{~min}$ at room temperature and followed by a solution of $10 \%$ normal goat serum (cat. no. S-1000; Vector Laboratories, Inc.) for $20 \mathrm{~min}$ at room temperature. These sections were reacted with a 1:1,000 dilution of rabbit anti-TNF- $\alpha$ (cat. no. ab66579, Abcam), a 1:200 dilution of rabbit anti-MCT4 (cat. no. ab244385, Abcam) overnight at $4^{\circ} \mathrm{C}$, and incubated in a 1:250 dilution of biotinylated goat anti-rabbit IgG (cat. no. BA-1000, Vector Laboratories, Inc.) for $2 \mathrm{~h}$ at room temperature and treated with a 1:200 dilution of streptavidin peroxidase complex (Vector Laboratories, Inc.) for $1 \mathrm{~h}$ at room temperature. Finally, these sections were visualized by reacting with a solution of 3,3'-diaminobenzidine tetrahydrochloride.

To quantitatively analyze TNF- $\alpha$ and MCT4 immunoreactivity, six sections with a $120-\mu \mathrm{m}$ interval per animal were selected. According to our previous method $(9,32)$, digital images of TNF- $\alpha$ and MCT4 immunoreactive structures were captured in the hippocampus with Axio Imager 2 microscope (Carl Zeiss AG) at magnification, $\mathrm{x} 20$ equipped with a digital camera. These images were calibrated into an array of 512 x 512 pixels. The density of MCT4 immunoreactive structures was evaluated as relative optical density (ROD) by using NIH ImageJ v1.59 software (National Institutes of Health,). A ratio of ROD was calibrated as \%, with the NT/control group (100\%).

Double immunofluorescence staining. To examine cell types containing TNF- $\alpha$ and MCT4 immunoreactivity, double immunofluorescence staining was performed according to our published protocol (33). In brief, rabbit anti-TNF- $\alpha$ (cat. no. ab66579, dilution 1:500; Abcam), goat anti-Ionized calcium binding adaptor molecule 1 (Iba1; cat. no. ab5076, dilution 1:400; Abcam) for microglia and rabbit anti-MCT4 (cat. no. ab244385, dilution 1:100; Abcam) and mouse anti-GFAP (cat. no. MAB360, dilution 1:400; Abcam) for astrocytes were used. The sections were incubated in the mixture of the antisera overnight at $4^{\circ} \mathrm{C}$, and the incubated sections were reacted in mixture of both donkey anti-rabbit IgG, Alexa Fluor488 (cat. no. A32790, dilution 1:500; Invitrogen; Thermo Fisher Scientific, Inc.) and goat anti-mouse IgG, Alexa Fluor546 (cat. no. A-11030, dilution 1:500; Invitrogen; Thermo Fisher Scientific, Inc.) and donkey anti-rabbit IgG, Alexa Fluor546 (cat. no. A10040, dilution 1:500; Invitrogen; Thermo Fisher Scientific, Inc.) and goat anti-mouse $\mathrm{IgG}$, or Alexa Fluor488 (cat. no. A-11001, dilution 1:500; Invitrogen; Thermo Fisher Scientific, Inc.) $2 \mathrm{~h}$ at room temperature. The immunoreaction was examined under confocal microscope (LSM510 META NLO; Carl Zeiss AG) at magnification, x20 in the Korea Basic Science Institute Chuncheon Center.

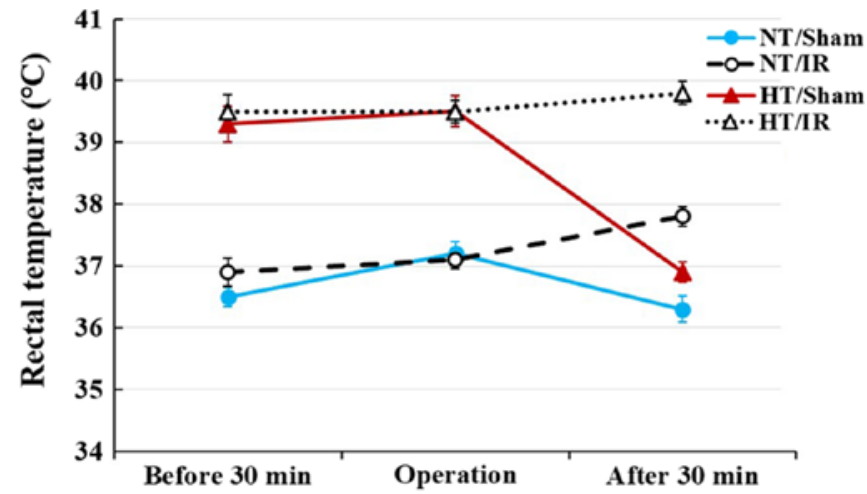

Figure 1. Change in rectal temperature in the NT/sham, NT/IR, HT/sham and $\mathrm{HT} / \mathrm{IR}$ groups for $30 \mathrm{~min}$ before and after IR. Normothermia $\left(37.0 \pm 0.2^{\circ} \mathrm{C}\right)$ or hyperthermia $\left(39.5 \pm 0.2^{\circ} \mathrm{C}\right)$ was controlled for $30 \mathrm{~min}$ before and during IR. Temperature in the NT/sham and HT/sham groups returned to normal body temperature at 30 min after sham IR; however, temperature in the NT/IR and HT/IR groups was slightly increased at $30 \mathrm{~min}$ after IR. The bars indicate the means \pm SEM ( $n=7$ per each group). IR, ischemia/reperfusion; NT, normothermia; HT, hyperthermia.

Statistical analysis. Data are expressed as the mean \pm SEM. Differences in the means among the groups were statistically analyzed by ANOVA with a post hoc Bonferroni's multiple comparison tests with SPSS v17.0 software (SPSS, Inc.). In order to elucidate ischemia-related differences among experimental groups. In order to compare two independent variables between normothermia and hyperthermia, and their interaction, two-way ANOVA was used with the Bonferroni post hoc. $\mathrm{P}<0.05$ was considered to indicate a statically significant difference.

\section{Results}

Change in body temperature. In the NT/sham group, rectal temperature was $36.5 \pm 0.2^{\circ} \mathrm{C}$ at $30 \mathrm{~min}$ before the sham operation, $37.2 \pm 0.2^{\circ} \mathrm{C}$ during the sham operation and $36.3 \pm 0.2^{\circ} \mathrm{C}$ at 30 min after sham operation (Fig. 1). In the NT/IR groups, rectal temperature was $36.9 \pm 0.2^{\circ} \mathrm{C}$ at $30 \mathrm{~min}$ before the IR operation, $37.1 \pm 0.1^{\circ} \mathrm{C}$ during the IR operation and $37.8 \pm 0.1^{\circ} \mathrm{C}$ at $30 \mathrm{~min}$ after the IR operation (Fig. 1).

In the HT/sham group, rectal temperature was $39.3 \pm 0.2^{\circ} \mathrm{C}$ at $30 \mathrm{~min}$ before the sham operation, maintained at $39.5 \pm 0.2^{\circ} \mathrm{C}$ during the sham operation and recovered to $36.9 \pm 0.1{ }^{\circ} \mathrm{C}$ (normal body temperature) at $30 \mathrm{~min}$ after the sham operation (Fig. 1). In the HT/IR groups, rectal temperature was $39.5 \pm 0.2^{\circ} \mathrm{C}$ at $30 \mathrm{~min}$ before the IR operation, $39.5 \pm 0.1^{\circ} \mathrm{C}$ during the IR operation and $39.8 \pm 0.1^{\circ} \mathrm{C}$ at 30 mins after the IR operation (Fig. 1).

IR-induced neuronal death. To examine IR-induced neuronal damage/death in the hippocampus, FJB (a marker for degenerating neurons) histofluorescence staining was performed. In the NT/control and NT/sham group, FJB-positive cells were not observed in CA1 (Fig. 2A, B and K). In the NT/IR groups, FJB-positive cells were not observed 2 days after IR (Fig. 2C and K). At 3 days after IR, a few FJB-positive degenerating cells were observed in the stratum pyramidale, in which pyramidal cells are located as principal cells in the hippocampus (Fig. 2D and K). At 5 days after IR, numerous FJB-positive degenerating cells were observed in the stratum 


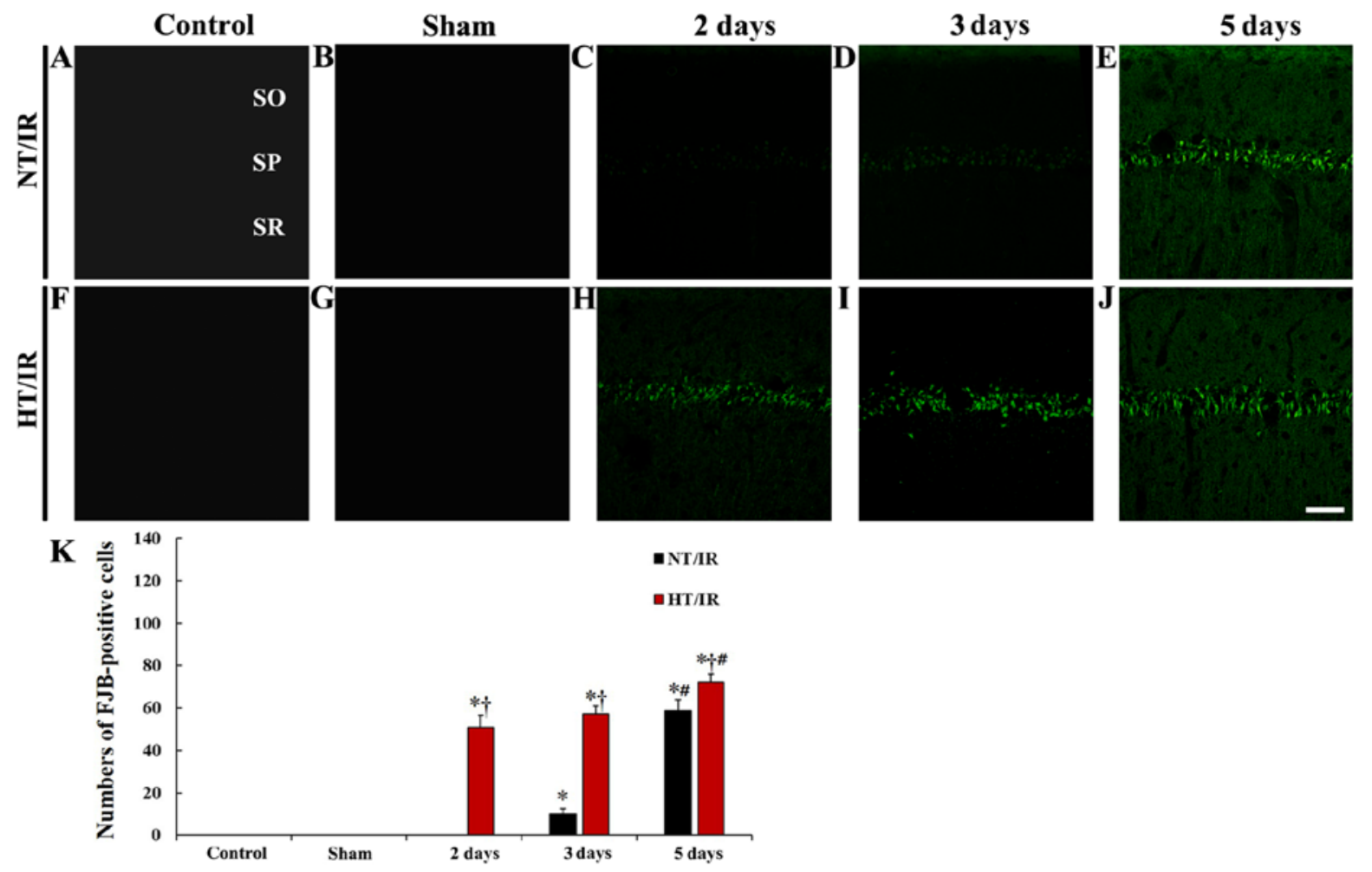

Figure 2. Fluoro-Jade B histofluorescence staining in CA1 of the (A) NT/control, (B) NT/sham, NT/IR at (C) 2, (D) 3 and (E) 5 days after IR, (F) HT/control, (G) HT/sham and HT/IR groups at (H) 2, (I) 3 and (J) 5 days after IR. In the NT/IR group, numerous FJB-positive cells were detected in the SP at 5 days after IR. However, in the HT/IR group, several FJB-positive cells were found from 2 days after IR. Scale bar, $50 \mu \mathrm{m}$. (K) Numbers of FJB-positive cells in CA1. ${ }^{~} \mathrm{P}<0.0001$ vs. NT/sham or HT/sham group; ${ }^{\dagger} \mathrm{P}<0.0001$, significantly different from the corresponding NT group; ${ }^{~} \mathrm{P}<0.0001$ vs. pre-time point group. The bars indicate the means \pm SEM. SO, stratum oriens; SR, stratum radiatum; CA1, cornu ammonis 1; IR, ischemia/reperfusion; NT, normothermia; HT, hyperthermia; SP, stratum pyramidale.

pyramidale, and the number of FJB-positive cells was significantly increased $(\mathrm{P}<0.0001)$ compared with that at 3 days after IR (Fig. 2E and K).

In the HT/control and HT/sham group, FJB-positive cells were not detected in any layers of CA1 (Fig. 2F, G and K). In the HT/IR groups, several FJB-positive cells began to be observed in the stratum pyramidale at 2 days after IR (Fig. $2 \mathrm{H}$ and $\mathrm{K}$ ). At 3 days after IR, the number of FJB-positive degenerating cells was increased to $112.1 \%$ compared to that at 2 days after IR (Fig. 2I and K), and, at 5 days after IR, FJB-positive degenerating cells were further increased $(\mathrm{P}=0.0035$; $141.2 \%$ compared to that at 2 days after IR) (Fig. 2J and K). In addition, at this point in time, the number of FJB-positive degenerating cells of the HT/IR group was significantly higher $(\mathrm{P}=0.024$, about $122.1 \%$ of the NT/IR group) than that in the NT/IR group (Fig. 2J and K).

IR-induced change in TNF- $\alpha$ immunoreactivity. In the NT/control and NT/sham groups, TNF- $\alpha$ immunoreactivity was observed in pyramidal neurons (Fig. 3A and B). In the NT/IR group, TNF- $\alpha$ immunoreactivity was significantly increased at $3 \mathrm{~h}(\mathrm{P}<0.0001)$ and $12 \mathrm{~h}(\mathrm{P}<0.0001)$ after $\mathrm{IR}$ and peaked at 1 day (337.7\% of the NT/sham group) after IR compared with the NT/sham group (Fig. 3C, D, E and Q). Thereafter, TNF- $\alpha$ immunoreactivity was gradually decreased at 2 days $(\mathrm{P}=0.0015)$ and 3 days $(\mathrm{P}<0.0001)$ after IR (Fig. 3F, $\mathrm{G}$ and $\mathrm{Q})$, and was barely observed at 5 days $(\mathrm{p}=0.0003)$ after IR (Fig. 3H) compared with the pre-time point group.
TNF- $\alpha$ immunoreactivity in the HT/control and HT/sham groups was observed in pyramidal neurons, and TNF- $\alpha$ immunoreactivity was significantly higher $(\mathrm{P}<0.0001)$ than that in the NT/control and NT/sham group (255.6 and $265.7 \%$, respectively) (Fig. 3I, J and Q). In the HT/IR group, TNF- $\alpha$ immunoreactivity was significantly increased from $3 \mathrm{~h} \quad(\mathrm{P}<0.0001) \quad(128.3 \%$ of the $\mathrm{HT} / \mathrm{sham}$ group) compared with the HT/sham group after IR and highest at $12 \mathrm{~h}(\mathrm{P}<0.0001)(221.4 \%$ of the $\mathrm{HT} / \mathrm{sham}$ group) after IR (Fig. 3K, L and Q). Thereafter, TNF- $\alpha$ immunoreactivity was significantly decreased at 1 day $(\mathrm{P}<0.0001)(124.3 \%$ of the HT/sham group) after IR compared to that at $12 \mathrm{~h}$ after ischemia and was barely observed at 2, 3 and 5 days after IR, showing that TNF- $\alpha$ immunoreactivity at 5 days after IR was increased in non-pyramidal cells of the strata oriens and radiatum (Fig. 3M-Q).

Double immunofluorescence staining results showed that, in the NT/IR and HT/IR groups, TNF- $\alpha$ immunoreactive non-pyramidal cells at 5 days after IR were merged with Iba-1 immunoreactive microglia (Fig. 3R).

IR-induced change in MCT4 immunoreactivity. MCT4 immunoreactivity in the NT/control and NT/sham groups was primarily observed in pyramidal neurons in CA1 (Fig. 4A and B). In the NT/IR group, MCT4 immunoreactivity in CA1 pyramidal neurons was significantly reduced at $3 \mathrm{~h}(\mathrm{P}<0.0001)$ and $12 \mathrm{~h}(\mathrm{P}<0.0001)$ after IR, compared to that in the NT/sham group (Fig. 4C, D and Q). MCT4 immu- 


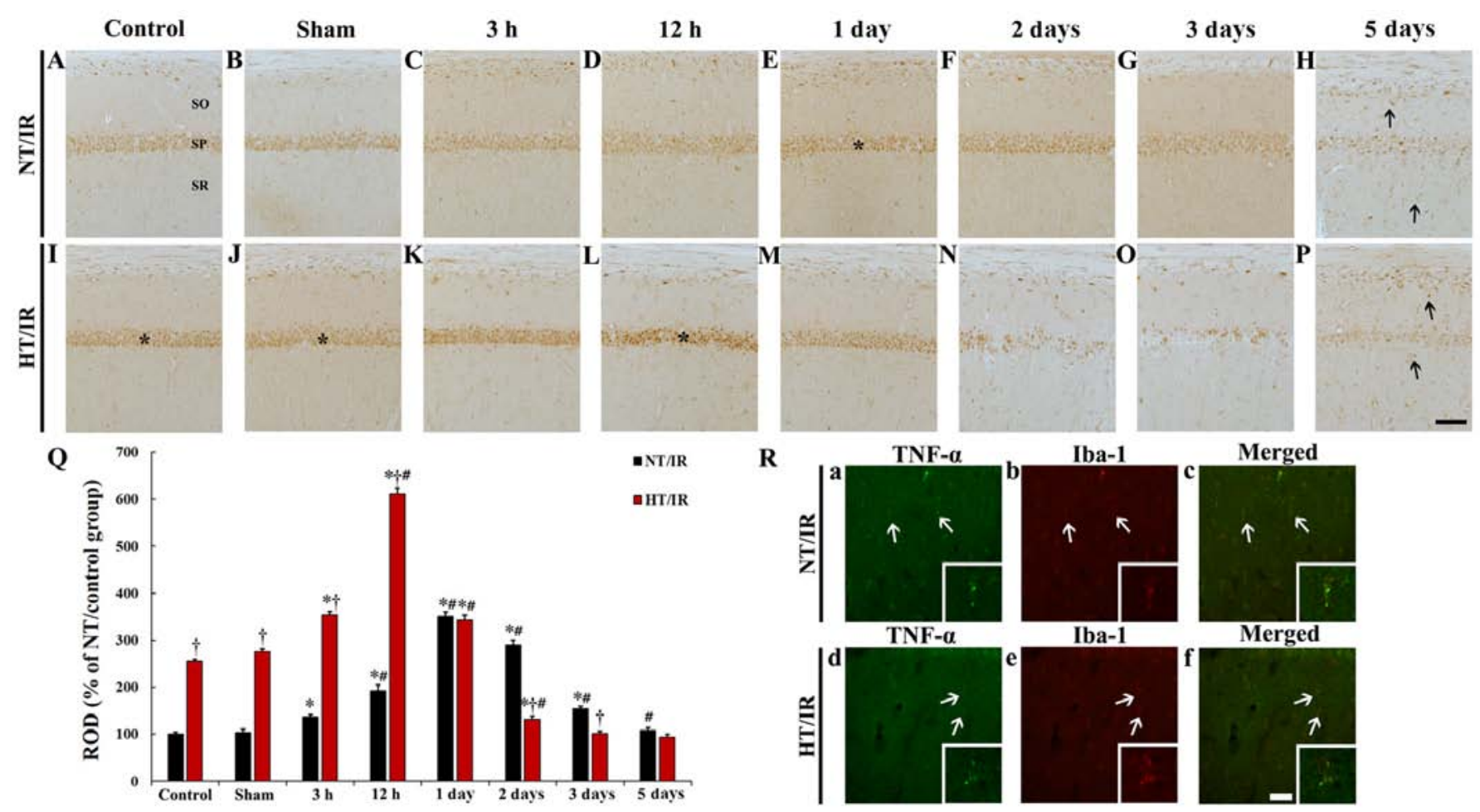

Figure 3. TNF- $\alpha$ immunohistochemistry in CA1 of the (A) NT/control, (B) NT/sham, NT/IR at (C) 3 h, (D) 12 h, (E) 1 day, (F) 2 days, (G) 3 days and (H) 5 days after IR, (I) HT/control, (J) HT/sham and HT/IR groups at (K) $3 \mathrm{~h}$, (L) $12 \mathrm{~h},(\mathrm{M}) 1$ day, (N) 2 days, (O) 3 days and (P) 5 days after IR. In the NT/IR group, TNF- $\alpha$ immunoreactivity increased gradually in the SP, peaked at 1 day post-IR and decreased thereafter. In the HT/control and HT/sham groups, TNF- $\alpha$ immunoreactivity in the SP was much higher (asterisks) than that in the NT/control and NT/sham groups. In the HT/IR group, TNF- $\alpha$ immunoreactivity increased significantly from $3 \mathrm{~h}$ post-IR, peaked at $12 \mathrm{~h}$ post-IR, decreased from 1 day post-IR and was barely observed at 3 and 5 days post-IR. Note that TNF- $\alpha$ immunoreactivity was observed in non-pyramidal cells (arrows) in SO and SR at 5 days after IR in both the NT/IR and HT/IR groups. Scale bar, $50 \mu \mathrm{m}$. (Q) ROD of TNF- $\alpha$ immunoreactivity as \% in CA1. " $\mathrm{P}<0.0001$, significantly different from the NT/sham or HT/sham group; ${ }^{\dagger} \mathrm{P}<0.0001$, significantly different from the corresponding NT group; ${ }^{~} \mathrm{P}<0.0001$ vs. pre-time point group. (R) Double immunofluorescence staining for TNF- $\alpha$ (green, a and d), Iba-1 (red, $b$ and e), and merged (c and f) images at 5 days post-IR in the NT/IR (upper panels) and the HT/IR (lower panels) groups. TNF- $\alpha$ immunoreactivity is merged with Iba-1 immunoreactive microglia (arrows). Scale bar, $50 \mu \mathrm{m}$. CA1, cornu ammonis 1; IR, ischemia/reperfusion; NT, normothermia; HT, hyperthermia; TNF, Tumor necrosis factor; SP, stratum pyramidale; SO, strata oriens; SR, stratum radiatum; ROD, Relative optical density; Iba, Ionized calcium binding adaptor molecule 1 .

noreactivity in the CA1 pyramidal neurons was significantly increased at 1 day $(\mathrm{P}<0.0001)$ and 2 days $(\mathrm{P}<0.0001)$ after IR compared to that in the NT/sham group (Fig. 4E, F and Q). At 3 and 5 days after IR, MCT4 immunoreactivity in the CA1 pyramidal neurons was barely observed compared with the NT/sham group, whereas MCT4 immunoreactivity was increased in non-pyramidal cells of the strata oriens and radiatum at 5 days after IR (Fig. 4G and $\mathrm{H}$ ).

In the HT/control and HT/sham group, no significant difference in MCT4 immunoreactivity in CA1 pyramidal neurons was shown, compared to that in the NT/control and NT/sham group (Fig. 4I, J and Q). At $3 \mathrm{~h}$ after IR, a significant increase $(\mathrm{P}<0.0001)(166.1 \%$ of the $\mathrm{HT} / \mathrm{sham}$ group) in MCT4 immunoreactivity was observed in the HT/IR group when compared with that in the HT/sham group, and the corresponding NT group (Fig. 4K and Q). MCT4 immunoreactivity in the CA1 pyramidal neurons in the HT/IR group was increased until 1 day after IR (Fig. 4L, M and Q). At 2 days after IR, only a few CA1 pyramidal neurons showed MCT4 immunoreactivity, and there was a significant decrease compared with the corresponding NT group (Fig. 4N). MCT4 immunoreactivity in the CA1 pyramidal neurons was barely observed at 3 days after IR and was significantly decreased compared with the corresponding NT group (Fig. 4O and Q). At 5 days after IR MCT4 immunoreactivity in CA1 pyramidal neurons was not observed but was expressed in non-pyramidal cells (Fig. 4P)

It was also found that MCT immunoreactivity in non-pyramidal cells at 5 days after IR in the NT/IR and HT/IR groups were identified as GFAP immunoreactive astrocytes (Fig. 4R).

\section{Discussion}

In the present study, body temperature of the NT/IR and HT/IR group was slightly increased $30 \mathrm{~min}$ after IR, while in the NT/sham and HT/sham group, body temperature was maintained after sham operation. Similar to the present results, it has been previously reported that cerebral ischemia raises body temperature $\left(38.5-39.5^{\circ} \mathrm{C}\right)$ until $\sim 1 \mathrm{~h}$ after IR, and then gradually recovers to normal body temperature in gerbils $(34,35)$. Therefore, the use of drugs to reduce body temperature immediately after ischemia can effectively protect neurons $(34,35)$. However, it has been reported that hyperthermia during the acute phase of cerebral ischemia within $24 \mathrm{~h}$ after IR exacerbates ischemic brain damage and worsens outcomes in patients with hyperthermia in acute 


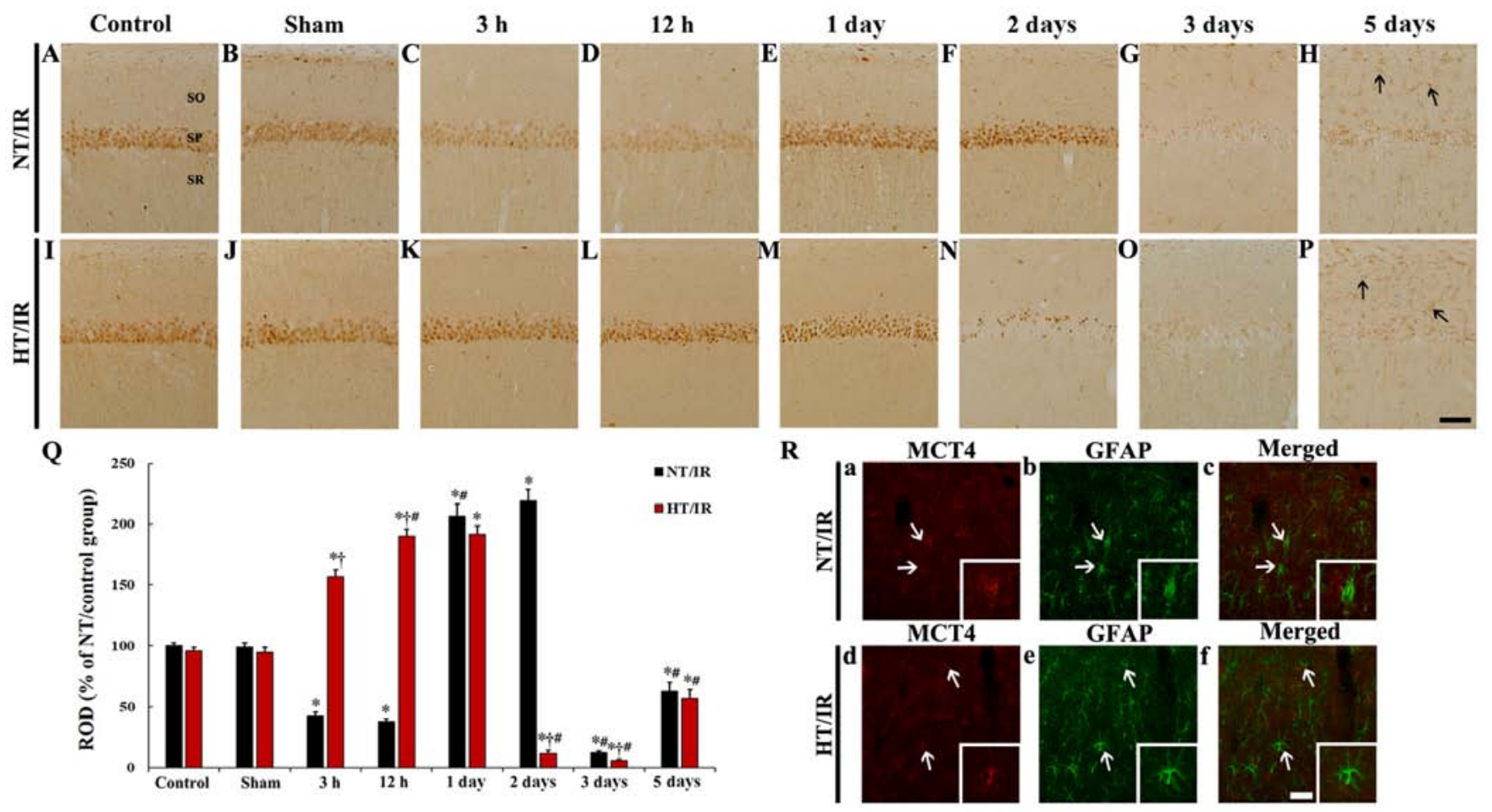

Figure 4. MCT4 immunohistochemistry in CA1 of the (A) NT/control, (B) NT/sham, NT/IR at (C) $3 \mathrm{~h}$, (D) $12 \mathrm{~h}$, (E) 1 day, (F) 2 days, (G) 3 days and (H) 5 days after IR, (I) HT/control, (J) HT/sham and HT/IR groups at (K) 3 h, (L) $12 \mathrm{~h},(\mathrm{M}) 1$ day, (N) 2 days, (O) 3 days and (P) 5 days after IR. In the NT/IR group, MCT4 immunoreactivity in the SP was markedly reduced at 3 and $12 \mathrm{~h}$ post-IR, significantly increased at 1 and 2 days post-IR, and barely observed at 3 and 5 days post-IR. In the HT/IR group, MCT4 immunoreactivity in the SP was significantly increased from $3 \mathrm{~h}$ post-IR, increased until 1 day post-IR, dramatically decreased at 2 days post-IR, and barely observed at 3 and 5 days post-IR. Note that MCT4 immunoreactivity was observed in non-pyramidal cells (arrows) in SO and SR at 5 days post-IR in both NT/IR and HT/IR groups. Scale bar, $50 \mu \mathrm{m}$. (Q) ROD of MCT4 immunoreactivity as \% in CA1. ${ }^{*} \mathrm{P}<0.0001$, significantly different from the NT/sham or HT/sham group; ${ }^{\circ} \mathrm{P}<0.0001$, significantly different from the corresponding NT group; ${ }^{\#} \mathrm{P}<0.0001$ vs. pre-time point group. (R) Double immunofluorescence staining for MCT4 (red, a and d), GFAP (green, b and e), and merged (c and f) images at 5 days post-IR in the NT/IR (upper panels) and the HT/IR (lower panels) groups. MCT4 immunoreactivity is merged with GFAP immunoreactive astrocytes (arrows). Scale bar, $50 \mu \mathrm{m}$. CA1, cornu ammonis 1; IR, ischemia/reperfusion; NT, normothermia; HT, hyperthermia; MCT4, Monocarboxylate transporter 4; SP, stratum pyramidale; SO, strata oriens; SR, stratum radiatum; ROD, Relative optical density.

ischemic stroke (36-38). Our previous studies revealed that hyperthermia before and during IR increased the extent and severity of IR-induced neuronal death and IR-induced glial activation in the gerbil hippocampus $(8,9)$. In the present study, it was found that IR-induced death of CA1 pyramidal neurons (principal neurons) was markedly augmented and occurred rapidly under hyperthermia when compared with that under normothermia. This finding was coincident with the result of our previous study (9).

There is extensive research on deleterious factors of hyperthermia in cerebral ischemic conditions, showing that induced hyperthermia can cause increases in oxidative stress and DNA fragmentation finally exacerbating neuronal damage in the hippocampus $(39,40)$. In addition, it has been reported that patients with hyperthermia have significantly higher plasma levels of TNF- $\alpha$ and increased infarct volume compared with the normothermic group, showing that there are significant correlations between TNF- $\alpha$ level and infarct volume, and between body temperature and infarct volume (37). Furthermore, brief hypoxia alone significantly increases brain TNF- $\alpha$ expression, and hyperthermia at $39^{\circ} \mathrm{C}$ following hypoxia causes a more significant increase in TNF- $\alpha$ expression in a rat model of perinatal inflammation (41). Similar to the results of the previous studies, in the present study, TNF- $\alpha$ immunoreactivity in CA1 pyramidal neurons located in the stratum pyramidale of the NT/IR group was gradually increased from $3 \mathrm{~h}$, peaked at 1 day and significantly decreased at 3 days after IR. Notably, hyperthermia without IR (HT/control and HT/sham group) significantly increased TNF- $\alpha$ immunoreactivity in CA1 pyramidal neurons, and in the HT/IR group, TNF- $\alpha$ immunoreactivity in the CA1 pyramidal neurons was much higher, rapidly increased and peaked at $12 \mathrm{~h}$ after IR compared with the NT/IR group. These results indicated that hyperthermia and IR under hyperthermia increases TNF- $\alpha$ expression (inflammatory response) in CA1 pyramidal neurons, showing that a significant increase in TNF- $\alpha$ expression under hyperthermia may be closely related to more severe ischemic damage to CA1 pyramidal neurons.

It has been suggested that MCT4 expression is closely related to TNF- $\alpha$ expression (42), indicating that the overexpression of MCT4 accelerates glycolysis, increases lactate (end product of glycolysis) and promotes pro-inflammatory cytokines in arsenite-induced liver carcinogenesis. Based on this report, the present study examined IR-induced changes in MCT4 immunoreactivity and found that the IR-induced changes in MCT4 immunoreactivity in CA1 pyramidal neurons under normothermia were significantly different from those under hyperthermia. 
It has been reported that MCT4 expression in the brain is altered after ischemic insults. For instance, MCT4 expression was found to be increased in the ipsilateral hemisphere at $1 \mathrm{~h}$ post-ischemia and then decreased at $24 \mathrm{~h}$ post-ischemia in a mouse model of transient middle cerebral artery occlusion (MCAO), which evoked focal brain ischemia (43). Another study showed that mRNA and protein expression levels of MCT4 and lactate levels were significantly increased in the rat brain after transient and permanent MCAO (44). In our previous and present studies, it was demonstrated that in CA1 region after IR under normothermia, MCT4 protein expression was decreased early after IR and markedly increased 1 and 2 days after IR (9). It has been reported that MCT4 is a high-capacity lactate transporter in cells exhibiting glycolytic activity and that MCT4 plays a crucial role in lactate release from glycolytic cells $(45,46)$. In addition, in a gerbil model of transient forebrain ischemia, the level of hippocampal lactate was significantly increased at $15 \mathrm{~min}$ after IR, distinctively decreased from $15 \mathrm{~min}$ to $6 \mathrm{~h}$ and similar to that in the control group at 2 days after IR (47). Collectively, it was indicated that IR-induced a decrease in MCT4 expression in CA1 pyramidal neurons soon after IR may be related to elevated usage of MCT4 to reduce the IR-induced increase in lactate levels. However, increases in MCT4 expression in CA1 pyramidal neurons at 1 and 2 days after IR may be associated with compensatory mechanisms (24).

In the present study on MCT4 expression in CA1 neurons of the HT/IR group, MCT4 immunoreactivity was notably increased from $3 \mathrm{~h}$ to 1 day after IR and markedly decreased at 2 days after IR, suggesting that the alteration pattern of MCT4 immunoreactivity was different from the NT/IR group. However, it is difficult to hypothesis the reasons as to why MCT4 protein expression in pyramidal neurons of CA1 induced by IR under hyperthermia was significantly increased soon after IR; this finding was different from that in pyramidal neurons of CA1 induced by IR under normothermia. Lactate produced by glia at post-ischemia is transported into neurons via MCTs for aerobic use, but inhibition of lactate transport into neurons via acting on MCTs ( $\alpha$-cyano-4-hydroxycinnamate, an MCT inhibitor) exacerbates neuronal damage in the rat hippocampus after IR induced by cardiac arrest (48). In addition, the alteration of MCT4 expression harms cellular survival after hypoxic exposure (23). Based on these studies and the present findings, it was suggested that a marked increase in MCT4 immunoreactivity in CA1 pyramidal neurons soon after IR under hyperthermia may be associated with increased glycolytic activities in the CA1 pyramidal neurons, and that the increase in MCT4 immunoreactivity at early points in time after IR may be related to the marked reduction in MCT4 immunoreactivity, as well as the advance of IR-induced death of CA1 pyramidal neurons from 2 days after IR.

However, there is a limitation to the present study. For instance, the present study examined IR-induced changes in TNF- $\alpha$ and MCT4 immunoreactivity in the CA1 pyramidal neurons, but did not investigate this using accurate quantitative experiments. Therefore, to clearly elucidate the roles and the expression changes in TNF- $\alpha$ and MCT4 following IR, quantitative analyses, such as western blot analysis or reverse transcription-quantitative PCR, should be performed in future studies.

In conclusion, IR under hyperthermia exacerbated the death of CA1 pyramidal neurons, suggesting that TNF- $\alpha$ and MCT4 immunoreactivity in the CA1 pyramidal neurons was high soon after IR under hyperthermia compared with that under normothermia. These results indicated that acceleration of neuronal death following IR under hyperthermia before and during IR may depend on the pattern of TNF- $\alpha$ and MCT4 protein expression after IR under hyperthermia.

\section{Acknowledgements}

Not applicable.

\section{Funding}

This study was supported by the National Research Foundation of Korea grant funded by the Korea government (MSIP; Ministry of Science, ICT \& Future Planning; grant no. NRF-2017R1C1B5075773) and by the Basic Science Research Program through the National Research Foundation of Korea funded by the Ministry of Education (grant no. NRF-2018R1D1A1B07049453).

\section{Availability of data and materials}

All data generated or analyzed during this study are included in this published article.

\section{Authors' contributions}

YEP, TKL and BK performed the experiments and measurements. JCL, JHC and JHP analyzed and interpreted the data. TGO, JHA, MHW and CHL made substantial contributions to conception and design of the research, and were involved in drafting, revising the manuscript and interpreting all data. All authors read and approved the manuscript, and agree to be accountable for all aspects of the research in ensuring that the accuracy or integrity of any part of the work are appropriately investigated and resolved.

\section{Ethics approval and consent to participate}

Experimental procedures for this study were approved by the Institutional Animal Care and Use Committee at Kangwon National University (approval no. KW-200113-1). The process of handling and caring animals conformed to the guidelines of the current international laws and policies (NIH Guide for the Care and Use of Laboratory Animals, The National Academies Press, 8th edition, 2011 and AVMA Guidelines for the Euthanasia of Animals, 2013 edition).

\section{Patient consent for publication}

Not applicable.

\section{Competing interests}

The authors declare that they have no competing interests. 


\section{References}

1. Kirino T: Delayed neuronal death in the gerbil hippocampus following ischemia. Brain Res 239: 57-69, 1982.

2. Onken M, Berger S and Kristian T: Simple model of forebrain ischemia in mouse. J Neurosci Methods 204: 254-261, 2012.

3. Lee CH, Yoo KY, Choi JH, Park OK, Hwang IK, Kim SK, Kang IJ, Kim YM and Won MH: Neuronal damage is much delayed and microgliosis is more severe in the aged hippocampus induced by transient cerebral ischemia compared to the adult hippocampus. J Neurol Sci 294: 1-6, 2010.

4. Liu F and McCullough LD: Interactions between age, sex, and hormones in experimental ischemic stroke. Neurochem Int 61: 1255-1265, 2012

5. Manwani B, Liu F, Scranton V, Hammond MD, Sansing LH and McCullough LD: Differential effects of aging and sex on stroke induced inflammation across the lifespan. Exp Neurol 249 120-131, 2013

6. Busto R, Dietrich WD, Globus MY and Ginsberg MD: The importance of brain temperature in cerebral ischemic injury. Stroke 20: 1113-1114, 1989.

7. Corbett D and Thornhill J: Temperature modulation (hypothermic and hyperthermic conditions) and its influence on histological and behavioral outcomes following cerebral ischemia. Brain Pathol 10: 145-152, 2000.

8. Kim DW, Cho JH, Cho GS, Kim IH, Park JH, Ahn JH, Chen BH, Shin BN, Tae HJ, Hong S, et al: Hyperthermic preconditioning severely accelerates neuronal damage in the gerbil ischemic hippocampal dentate gyrus via decreasing SODs expressions. J Neurol Sci 358: 266-275, 2015.

9. Kim MJ, Cho JH, Cho JH, Park JH, Ahn JH, Tae HJ, Cho GS, Yan BC, Hwang IK, Lee CH, et al: Impact of hyperthermia before and during ischemia-reperfusion on neuronal damage and gliosis in the gerbil hippocampus induced by transient cerebral ischemia. J Neurol Sci 348: 101-110, 2015.

10. Barber PA, Hoyte L, Colbourne F and Buchan AM Temperature-regulated model of focal ischemia in the mouse: A study with histopathological and behavioral outcomes. Stroke 35 1720-1725, 2004.

11. Campos F, Blanco M, Barral D, Agulla J, Ramos-Cabrer P and Castillo J: Influence of temperature on ischemic brain: Basic and clinical principles. Neurochem Int 60: 495-505, 2012.

12. Kim Y, Busto R, Dietrich WD, Kraydieh S and Ginsberg MD Delayed postischemic hyperthermia in awake rats worsens the histopathological outcome of transient focal cerebral ischemia. Stroke 27: 2274-2280, discussion 2281, 1996.

13. Satoh K, Niwa M, Binh NH, Nakashima M, Kobayashi K, Takamatsu M and Hara A: Increase of galectin-3 expression in microglia by hyperthermia in delayed neuronal death of hippocampal CA1 following transient forebrain ischemia. Neurosci Lett 504: 199-203, 2011

14. Wang CX, Stroink A, Casto JM and Kattner K: Hyperthermia exacerbates ischaemic brain injury. Int J Stroke 4: 274-284, 2009.

15. Saxton C: Effects of severe heat stress on respiration and metabolic rate in resting man. Aviat Space Environ Med 52: 281-286, 1981.

16. Wood SC and Gonzales R: Hypothermia in hypoxic animals: Mechanisms, mediators, and functional significance. Comp Biochem Physiol B Biochem Mol Biol 113: 37-43, 1996.

17. Arnberg F, Grafström J, Lundberg J, Nikkhou-Aski S, Little P, Damberg P, Mitsios N, Mulder J, Lu L, Söderman M, et al: Imaging of a clinically relevant stroke model: Glucose hypermetabolism revisited. Stroke 46: 835-842, 2015

18. Karaszewski B, Wardlaw JM, Marshall I, Cvoro V, Wartolowska K Haga K, Armitage PA, Bastin ME and Dennis MS: Early brain temperature elevation and anaerobic metabolism in human acute ischaemic stroke. Brain 132: 955-964, 2009.

19. Schurr A: Lactate, glucose and energy metabolism in the ischemic brain (Review). Int J Mol Med 10: 131-136, 2002. (Review).

20. Pierre K and Pellerin L: Monocarboxylate transporters in the central nervous system: Distribution, regulation and function. J Neurochem 94: 1-14, 2005.

21. Hong S, Ahn JY, Cho GS, Kim IH, Cho JH, Ahn JH, Park JH, Won MH, Chen BH, Shin BN, et al: Monocarboxylate transporter 4 plays a significant role in the neuroprotective mechanism of ischemic preconditioning in transient cerebral ischemia. Neural Regen Res 10: 1604-1611, 2015.

22. Pellerin L, Bergersen LH, Halestrap AP and Pierre K: Cellular and subcellular distribution of monocarboxylate transporters in cultured brain cells and in the adult brain. J Neurosci Res 79: $55-64,2005$
23. Rosafio K and Pellerin L: Oxygen tension controls the expression of the monocarboxylate transporter MCT4 in cultured mouse cortical astrocytes via a hypoxia-inducible factor- $1 \alpha$-mediated transcriptional regulation. Glia 62: 477-490, 2014.

24. Yoo DY, Park JH, Lee KY, Kwon HJ, Jung HY, Kim JW, Kim DW, Choi JH, Moon SM, Yoon YS, et al: Temporal and spatial changes of monocarboxylate transporter 4 expression in the hippocampal CA1 region following transient forebrain ischemia in the Mongolian gerbil. Mol Med Rep 15: 4225-4230, 2017.

25. Council NR: Guide for the Care and Use of Laboratory Animals. National Academies Press. Washington, DC, 2010.

26. Leary SL, Underwood W, Anthony R, Cartner S, Corey D, Grandin T, Greenacre C, Gwaltney-Brant S, McCrackin M and Meyer R: AVMA guidelines for the euthanasia of animals. 1st edition. American Veterinary Medical Association. Schaumburg, IL, 2013.

27. Kaufman GD, Shinder ME and Perachio AA: Correlation of Fos expression and circling asymmetry during gerbil vestibular compensation. Brain Res 817: 246-255, 1999.

28. Du X, Wang D, Li Y, Huo X, Li C, Lu J, Wang Y, Guo M and Chen Z: Newly breeding an inbred strain of ischemia-prone Mongolian gerbils and its reproduction and genetic characteristics. Exp Anim 67: 83-90, 2018

29. Zhu X, Yan B,Tang C, Qiu G,Wu Y,Wang J and BoP:Neuroprotective effect of Paeoniae Radix Rubra on hippocampal CA1 region of mice induced by transient focal cerebral ischemia via anti-gliosis and anti-oxidant activity. Chin Herb Med 11: 86-91, 2019.

30. Diven K: Inhalation anesthetics in rodents. Lab Anim (NY) 32: 44-47, 2003.

31. Carpenter JW: Exotic Animal Formulary-eBook. 4th edition. Elsevier Health Sciences. Amsterdam, Netherlands, 2012.

32. Ahn JH, Kim DW, Park JH, Lee TK, Lee HA, Won MH and Lee CH: Expression changes of CX3CL1 and CX3CR1 proteins in the hippocampal CA1 field of the gerbil following transient global cerebral ischemia. Int J Mol Med 44: 939-948, 2019.

33. Park JH, Shin BN, Ahn JH, Cho JH, Kim IH, Kim DW, Won MH, Hong S, Cho JH and Lee CH: Ischemia-Induced Changes of PRAS40 and p-PRAS40 Immunoreactivities in the gerbil hippocampal CA1 region after transient cerebral ischemia. Cell Mol Neurobiol 36: 821-828, 2016

34. Won MH, Lee JC, Kim YH, Song DK, Suh HW, Oh YS, Kim JH, Shin TK, Lee YJ and Wie MB: Postischemic hypothermia induced by eugenol protects hippocampal neurons from global ischemia in gerbils. Neurosci Lett 254: 101-104, 1998.

35. Yang GE, Tae H-J, Lee T-K, Park YE, Cho JH, Kim DW, Park $\mathrm{JH}, \mathrm{Ahn} \mathrm{JH}$, Ryoo S, Kim Y-M, et al: Risperidone treatment after transient ischemia induces hypothermia and provides neuroprotection in the gerbil hippocampus by decreasing oxidative stress. Int J Mol Sci 20: 4621, 2019

36. Zaremba J: Hyperthermia in ischemic stroke. Med Sci Monit 10: RA148-RA153, 2004

37. Leira R, Rodríguez-Yáñez M, Castellanos M, Blanco M, Nombela F, Sobrino T, Lizasoain I, Dávalos A and Castillo J: Hyperthermia is a surrogate marker of inflammation-mediated cause of brain damage in acute ischaemic stroke. J Intern Med 260: 343-349, 2006

38. de Jonge JC, Wallet J and van der Worp HB: Fever worsens outcomes in animal models of ischaemic stroke: A systematic review and meta-analysis. Eur Stroke J 4: 29-38, 2019.

39. Hara A, Niwa M, Iwai T, Yano H, Bunai Y,Uematsu T, Yoshimi N and Mori H: Increase of fragmented DNA transport in apical dendrites of gerbil CA1 pyramidal neurons following transient forebrain ischemia by mild hypothermia. Neurosci Lett 280: 73-77, 2000.

40. Kil HY, Zhang J and Piantadosi CA: Brain temperature alters hydroxyl radical production during cerebral ischemia/reperfusion in rats. J Cereb Blood Flow Metab 16: 100-106, 1996.

41. Wang W, Dow KE and Flavin MP: Hyperthermia amplifies brain cytokine and reactive oxygen species response in a model of perinatal inflammation. Neurosci Lett 445: 233-235, 2008.

42. Luo F, Zou Z, Liu X, Ling M, Wang Q, Wang Q, Lu L, Shi L, Liu Y, Liu Q, et al: Enhanced glycolysis, regulated by HIF-1a via MCT-4, promotes inflammation in arsenite-induced carcinogenesis. Carcinogenesis 38: 615-626, 2017.

43. Rosafio K, Castillo X, Hirt L and Pellerin L: Cell-specific modulation of monocarboxylate transporter expression contributes to the metabolic reprograming taking place following cerebral ischemia. Neuroscience 317: 108-120, 2016

44. Geng X, Sy CA, Kwiecien TD, Ji X, Peng C, Rastogi R, Cai L, Du H, Brogan D, Singh S, et al: Reduced cerebral monocarboxylate transporters and lactate levels by ethanol and normobaric oxygen therapy in severe transient and permanent ischemic stroke. Brain Res 1603: 65-75, 2015. 
45. Dimmer KS, Friedrich B, Lang F, Deitmer JW and Bröer S: The low-affinity monocarboxylate transporter MCT4 is adapted to the export of lactate in highly glycolytic cells. Biochem J 350: 219-227, 2000

46. Halestrap AP and Meredith D: The SLC16 gene family-from monocarboxylate transporters (MCTs) to aromatic amino acid transporters and beyond. Pflugers Arch 447: 619-628, 2004.

47. Kim W, Kwon HJ, Jung HY, Yoo DY, Kim DW and Hwang IK: Phosphoglycerate mutase 1 reduces neuronal damage in the hippocampus following ischemia/reperfusion through the facilitation of energy utilization. Neurochem Int 133: 104631, 2020.
48. Schurr A, Payne RS, Miller JJ, Tseng MT and Rigor BM: Blockade of lactate transport exacerbates delayed neuronal damage in a rat model of cerebral ischemia. Brain Res 895: 268-272, 2001.

(i) (9) This work is licensed under a Creative Commons EY NC ND Attribution-NonCommercial-NoDerivatives 4.0 International (CC BY-NC-ND 4.0) License. 